\title{
Development of Model for Extrusion Cooking Based on Textural Properties of Extrudate Prepared from Rice Broken and Pigeon Pea Dal Broken Flour Blends
}

\author{
Ajeet Sarathe $^{1 *}$, Ashwani Duggal ${ }^{2}$ and Mohan Singh $^{3}$ \\ ${ }^{1}$ Department of Agricultural Engineering, Faculty of Agri. Sci. \& Tech., AKS University, \\ Satna (M.P.), 485001, India \\ ${ }^{2}$ Department of Food and Agriculture Engineering, MGCGV Chitrakoot (MP) \\ ${ }^{3}$ Department of Post Harvest Process \& Food Engineering, College of Agricultural \\ Engineering, JNKVV Jabalpur (M.P.), 482004 \\ *Corresponding author
}

\section{A B S T R A C T}

\begin{tabular}{|l|}
\hline K e y w o r d s \\
Extrusion cooking, \\
Textural properties, \\
Hardness, Cutting \\
strength, Response \\
surface \\
methodology, \\
model, By-product \\
utilization
\end{tabular}

\section{Keywords}

Extrusion cooking,

Textural properties,

Hardness, Cutting

surface

methodology,

model, By-product

utilization

17 September 2019

10 October 2019
The traditional milling processing practices used in rice and pulses milling, yields significant amounts in the form of brokens from rice and dal mill industries. That brokens do not find appropriate market and are listed as losses of the milling industries, generally disposed off cheaply, whereas they are equally rich in the nutrition as comparable to whole grain. Present study has been conducted with the aim of fruitful utilization of by-product of rice and dal mill industries i.e. brokens by using extrusion cooking technology to prepare snacks by using a Brabender Single - Screw laboratory extruder. The effect of moisture content, feed blend ratio, barrel temperature and screw speed of extruder on textural characteristics of extrudates were studied by using Texture Analyzer. A central composite rotatable design of Response surface methodology was used to develop prediction model. Second order quadratic regression model was fitted in the variation. The significance was established at $\mathrm{p} \leq 0.05$. The hardness of extrudates varied from $4.63 \mathrm{~N}$ and $22.30 \mathrm{~N}$ whereas the cutting strength of extrudates varied from $4.07 \mathrm{~kg}$ to $15.86 \mathrm{~kg}$. It was observed that moisture content and blend ratio of feed, and barrel temperature gave significant role of textural characteristics of extrudates.

\section{Introduction}

Extrusion is one of the most dynamic, versatile, and well-established industrial processes used in the food and feed industry today. It is being extensively used worldwide to produce an ever-expanding list of food and feed products including snacks, cereals, pastas, TVPs (texturized vegetable proteins), pet foods, animal feeds, instant beverages, meat analogs, and a range of ethnic foods (Shah, 2003). India has indeed a very good potential demand for cereals and pulses based ready-to-eat soy fortified extruded snacks. Extrusion technologies are used for cereal and protein processing in the food and closely 
related pet foods and feed sectors. Extrusion has become an important processing technique and its application today cover a wide range of food products based on starch cereals protein and sugar. Now-a-days extrusion cooking technology is one of a most significance manufacturing tool for food and feed processing industries. The significance of it should not be underestimated since the technology is relatively new as well as during the last some years it is one of the successful unit operations for food processing industries in India that is still developing rapidly. India has indeed a very good potential demand for cereals and pulses based ready-to-eat extruded snacks. Extrusion cooking has advantages, including versatility, high productivity, low operating costs, energy efficiency, and shorter cooking times (Harper 1981). Several legumes have been treated by extrusion and good expansion has been reported (Balandran et al 1998). In addition, and as a result of high temperatures, high pressures, and several shear forces reached inside the barrel, chemical reactions and molecular modifications like gelatinization of starch, denaturation of proteins, inactivation of many food enzymes, and reduction of microbial counts can occur (Harper, 1981). Inside the extruder the cereal mixture is heated above the starch gelatinization temperature leading to a cooked product that may be directly enrobed and flavoured, or may need further processing such as frying or roasting. In the blend of flours made from starch and protein rich grains, the selection of machine and process parameters for extrusion becomes more important as the starch gets gelatinized and protein gets denatured at different process parameters. Hence a consorted effort is required to get optimum physical properties of the extrudate making to be more acceptable to the consumer. Product quality can vary considerably depending on the extruder type, screw configuration, feed moisture, and temperature profile in the barrel session, screw speed and feed rate (Ding et al., 2005). In fact, the chemical and physical characteristics of products strongly depend upon process variables such as extrusion temperature, screw speed and moisture content (De Cindio et al., 2002). In cereal-based products, the degree or proper processing of starch is important for major quality aspects such as taste, digestibility, texture, appearance and puffing. Extrusion operational parameters such as barrel temperature, and screw speed affect the snack quality. In addition to these, processing parameters like feed moisture content, blending ratio also play important role on the quality of extrudate. Therefore, the effects of various operational and processing parameters and their interaction on quality of extrudate have to be studied and established. Present study has been conducted to investigate and standardized various operational and process parameters and their interaction on textural quality of extrudated snacks prepared from byproducts of rice and dal milling industries i.e. brokens. Simultaneously, models have been developed, which could be used to optimize parameters for best textural quality of readyto-eat snack food product.

\section{Materials and Methods}

The raw material for the study i.e. rice and dal broken were procured from Department of Agricultural Engineering, AKS University, Satna (M.P.). After initial removal of foreign materials, flour was prepared by grinding in the hammer mill. The moisture content of flour at different blend ratio was then measured by standard oven drying method. After getting the moisture content of rice broken flour and dal broken flour blends, additional water required to raise the moisture content to desired levels of blends i.e. 9, 12, 15,18 and $21 \%$ (wb) was calculated. Then calculated amount of water plus an additional $10 \%$ of calculated water was added to supplement the evaporation losses during 
mixing and conditioning. Tempering of samples was done by keeping the moistened samples for $24 \mathrm{~h}$ at room temperature $\left(25^{\circ} \mathrm{C}\right)$ so as to get uniform distribution of moisture throughout the mass of blends. The conditioned sample was then feed to the laboratory-scale single-screw extruder (model Kompakt E-19/25 D Brabender Duisburg, Germaney ) at pre set operational conditions (length-to-dia 20:1; compression ratio 2:1 and die opening $5 \mathrm{~mm}$ ) for extrusion cooking. The product after coming out of the extruder discharge end through round die, expanded due to sudden release of pressure. The extrudates were collected and packed in laminated polythene bags and properly labelled for the analysis purpose.

\section{Experiment design and analysis}

Design experiments were conducted following Response Surface Methodology (RSM) (Myres, 1976). This is a combination of mathematical and statistical techniques that are useful for the modeling and analysis of problems, in which response of interest is influenced by several variables and objective is to optimize the response. In the present study, Central Composite Rotatable Design (CCRD) of five independent variables with five level of each was used. In this study the effect of processing parameters i.e. bland ratio of rice broken flour and dal broken flour (90:10, 80:20, 70:30, 60:40 and 50:50), moisture content (w.b.) of feed (9, 12, 15, 18 and $21 \%$ ), die head temperature $(180,190$, 200, 210 and $\left.220{ }^{\circ} \mathrm{C}\right)$, barrel temperature (120, $125,130,135$ and $140{ }^{\circ} \mathrm{C}$ ) and screw speed $(120,125,130,135$ and $140 \mathrm{rpm})$ was optimized against the following textural quality responses i.e. hardness, crispness and cutting strength of extrudates. Prediction models were developed for each of the responses, followed by the model analysis which included checking the validity of the model with the help of various prescribed statistical aids. Optimum values were obtained for all the processing variables by keeping the response either in range at minimum or at maximum.

Data analysis was done for optimization of operational and processing parameters of extruder on textural properties of extrudates prepared out of rice broken flour and dal broken flour blends using software, Design expert 11.1.2.0 (32bit). Based on the executed statistics, such as p-values, lack of fit and $\mathrm{R}^{2}$, PRESS values for comparing the models, software executed statistics and suggested linear and Quadratic model significant at $\mathrm{p}<0.0001$ with $\mathrm{R}^{2}=0.999$ and $\mathrm{R}^{2}=0.975$, lowest PRESS and insignificant lack of fit was selected. Coefficients of the model were estimated for each response. Best-fit regression equations were also developed. Response surface graphs were drawn with the help of Design expert 11.1.2.0 to know the effect of independent variables on the responses. The observed data was analyzed, employing multiple regression technique. The best fitting model was chosen, based on lack of fit criteria (Cochran and Cox 1957).

\section{Determination of textural properties}

A Texture Analyzer TA - XT2 $i$ available at Department of Post Harvest Process and Food Engineering, CAE, Jabalpur is a highly scientific device was used to study the crispness, hardness and cutting strength of extrudates.

\section{Results and Discussion}

The data obtained from the experiments for different combinations (Table 2) were analyzed by using multiple regression and second order polynomial model and fitted to the experimental data with coded values of independent variables and inter- treated with the help of models and graphs. 
From the tabulated values, three-dimensional graphs were prepared treating two independent variables to be constant and showing the effect of other two variables on textural properties i.e. crispness, hardness and cutting strength of extrudates. Adequacy of the model was tested using Fisher's F-test. The significance was established at $\mathrm{p} \leq 0.05$. The effects of variables have been interpreted. The findings have been explained suitably with logical reasons wherever possible. The findings also have been discussed in the light of theories and with the literature support to the possible extent. The process parameters considered were moisture content and blend ratio of feed where as the operational parameters were barrel temperature, die head temperature and screw speed. The experimental details are given in Table-2.

\section{Effect of process and operational parameters on crispness of extrudate}

Crispiness is the most important texture attribute which attracts the consumer's preference. Crispiness is measured as fractures by texture analyzer and values ranged from 11- 22 fractures. The $\mathrm{R}^{2}$ had a value of 0.7941 for the model. The results of analysis of variance (ANOVA) for model are presented in Table-3 and regression coefficient and standard error of second order mathematical model are reported in Table- 6 the significance of each terms are also reported. The positive coefficient at linear level indicated that there was increase in response with increase in level of selected parameters and vice versa. Negative quadratic terms indicate that the maximum value of the response was obtained at the centre point while positive quadratic term gave the minimum response.

Using CCRD and fitting of second degree polynomial equation for representative response surface of data resulted in the development of following model;
Crispness $=-571.464-0.941692 \times$ FMC $0.708455 \times \mathrm{BR}+1.20834 \times \mathrm{DHT}+4.3367 \times$ $\mathrm{BT}+3.01754 \times \mathrm{SS}+0.00841667 \times \mathrm{FMC} \times$ $\mathrm{BR}+0.004625 \times$ FMC $\times$ DHT $-0.0143333 \times$ FMC $\times$ BT + $0.0101667 \times$ FMC $\times$ SS + $0.003025 \times \mathrm{BR} \times \mathrm{DHT}+0.005425 \times \mathrm{BR} \times \mathrm{BT}$ $-0.002225 \times$ BR $\times$ SS $-0.0053 \times$ DHT $\times$ BT + $0.00105 \times$ DHT $\times \mathrm{SS}+0.00585 \times \mathrm{BT} \times \mathrm{SS}+$ 0.00823232 x FMC2 - 0.00875909 x BR2 $0.00200909 \times$ DHT2 - $0.0150364 \times$ BT2 $0.0155864 \times \mathrm{SS} 2$

It is observed that crispness increases with decrease in moisture content of feed as an indicative of creation of more porous structure of extrudates leading to increased crispness. It has also observed that, the crispness of extrudates increases, with decrease in percent of dal broken flour, it contain higher amount of protein and fiber than rice broken flour. It is evident that with increase in barrel temperature crispness of extrudates increases, however, die head temperature had less effect on the crispness of the extrudates. The crispness of the extrudates varied between 11.31 to 16.66. Effects of process and operational parameters on extrudate were shown in Figures 1-10. Maximum value of crispness of extrudates was observed at $21 \%$ moisture content of feed, 70:30 of blend ratio, $130{ }^{0} \mathrm{C}$ barrel temperature, $200{ }^{0} \mathrm{C}$ die head temperature and $130 \mathrm{rpm}$ screw speed.

\section{Effect of process and operational parameters on hardness of extrudate}

Texture is the ultimate criteria on which acceptability of any snack depends and hardness is one important attribute to express texture. Hardness was measured by compression force using the texture analyzer. The multiple regression analysis for hardness of extrudates versus feed moisture content (FMC), blend ratio (RB), barrel temperature (BT), die head temperature (DHT) and screw speed (SS) was done using CCRD and fitting of second degree polynomial equation for 
representative response surface of data resulted in the development of following model;

Hardness $=165.149+10.6963 \times \mathrm{FMC}+$ $8.62283 * \mathrm{x}$ BR + 1.93636 x DHT -3.42295 x BT $-4.99395 \times$ SS -0.0279583 x FMC x BR $+0.0167917 \times$ FMC $x$ DHT $-0.14325 \times$ FMC $\mathrm{x}$ BT + $0.0455 \times$ FMC $x$ SS -0.0256875 x BR x DHT - 0.018125 x BR x BT -0.00715 x BR x SS $-0.010975 \times$ DHT $x$ BT $-0.00375 \times$ DHT $x$ SS $-0.0048 \times$ BT $x$ SS $-0.0158712 \times$ $\mathrm{FMC}^{2}+0.00430909 \times \mathrm{BR}^{2}+0.00114659 \mathrm{x}$ $\mathrm{DHT}^{2}+0.0327864 \mathrm{x} \mathrm{BT}^{2}+0.0237864 \mathrm{x}$ $\mathrm{SS}^{2}$ (2)

The $\mathrm{R}^{2}$ had a value of 0.7280 for the model. The results of analysis of variance (ANOVA) for model 2 are presented in Table- 4 .

The F-value 1.47 implies that the model is non-significant. Regression coefficients of second order mathematical model are reported in Table-7, the significance of each terms is also reported.

Hardness of extrudate is the resistance offered to breaking when subjected to compressive loading. The hardness of the extrudates varied between 4.63 and $22.30 \mathrm{~N}$. As clearly seen from Fig. 11 the hardness of extrudates reduces with decrease in blend ratio of dal broken flour and has almost no effect with change in barrel temperature and die head temperature and slight increase with increase in screw speed. Hardness of extrudate increases with increase in blend ratio of dal broken flour this may be because the dough of dal broken flour constitutes a hard mass once it is dried after melting which is mainly due to the characteristics of protein present in dal broken flour.

As it is seen from Fig. 13 that hardness reduces with increase in die head temperature, which may be mainly due to the changing of protein and fibre content of dal broken flour at higher temperature. Increase in screw speed creates a better homogenous mass by better shearing of melt inside the barrel which creates a uniform porous structure of extrudate imparting a soft upper coat to the extrudate. Increase in temperature decreased the hardness which is in agreement with the result of rice and green gram extrudates (Bhattacharya, 1997). Higher temperature leads to expansion of extrudates as the superheated steam is released and disrupts the material due to shear which results in lighter extrudates with less hardness. As moisture content increased hardness increased which is in agreement with the results of Ding et.al., (2006) where wheat based expanded snacks were studied.

Effect of process and operational parameters on cutting strength of extrudate

The multiple regression analysis for cutting strength of extrudates versus feed moisture content (FMC), blend ratio (RB), barrel temperature (BT), die head temperature (DHT) and screw speed (SS) was done using CCRD and fitting of second degree polynomial equation for representative response surface resulted in the development of following model;

Cutting strength $=-204.902-14.732 \mathrm{x}$ FMC $-0.212967 \times$ BR + $1.39705 \times$ DHT + $1.13957 \times \mathrm{BT}+1.58117 \times \mathrm{SS}+0.0243958 \times$ FMC $\times$ BR + $0.0135625 \times$ FMC $\times$ DHT + 0.0654583 x FMC x BT + 0.00729167 x FMC $\mathrm{x}$ SS + $0.00215625 \times$ BR $\times$ DHT -0.0168875 $\mathrm{x}$ BR $\times$ BT $+0.0127125 \times$ BR $\times$ SS + 0.0023625 x DHT x BT -0.0097875 x DHT x $\mathrm{SS}+0.042425 \times \mathrm{BT} \times \mathrm{SS}+0.0719268 \times$ $\mathrm{FMC}^{2}+0.00218591 \times \mathrm{BR}^{2}-0.00171409 \mathrm{x}$ $\mathrm{DHT}^{2}-0.0288564 \times \mathrm{BT}^{2}-0.0217464 \times \mathrm{SS}^{2}$

......(3)

The $\mathrm{R}^{2}$ had a value of 0.7814 for the model. The results of analysis of variance (ANOVA) 
for model 3 are presented in Table-5 and mathematical model are reported in Table-8. regression coefficients of second order

Table.1 Level of coded variables

\begin{tabular}{|l|c|c|c|c|c|}
\hline Independent variable & \multicolumn{4}{|c|}{ Code levels } \\
\cline { 2 - 5 } & $\mathbf{- 2}$ & $\mathbf{- 1}$ & $\mathbf{0}$ & $\mathbf{1}$ & $\mathbf{2}$ \\
\hline Moisture Content (\% w.b.) & 9 & 12 & 15 & 18 & $\mathbf{2 1}$ \\
\hline Blend ratio & $90: 10$ & $80: 20$ & $70: 30$ & $60: 40$ & $\mathbf{5 0 : 5 0}$ \\
\hline Barrel Temperature $\left({ }^{\mathbf{0}} \mathbf{C}\right)$ & 120 & 125 & 130 & 135 & $\mathbf{1 4 0}$ \\
\hline Die Head Temperature $\left({ }^{\mathbf{0}} \mathbf{C}\right)$ & 180 & 190 & 200 & 210 & $\mathbf{2 2 0}$ \\
\hline Screw Speed $(\mathbf{r p m})$ & $\mathbf{1 2 0}$ & $\mathbf{1 2 5}$ & $\mathbf{1 3 0}$ & $\mathbf{1 3 5}$ & $\mathbf{1 4 0}$ \\
\hline
\end{tabular}

Table.2 Experimental design matrix for parameter levels

\begin{tabular}{|c|c|c|c|c|c|c|c|c|c|c|}
\hline \multirow[t]{3}{*}{ Run } & \multicolumn{5}{|c|}{ Coded Values } & \multicolumn{5}{|c|}{ Actual Values } \\
\hline & F - 1 & F - 2 & F - 3 & F -4 & F -5 & F - 1 & F - 2 & F -3 & F -4 & F - 5 \\
\hline & $\mathbf{A}$ & B & $\mathbf{C}$ & D & $\mathbf{E}$ & $\mathbf{A}$ & B & C & D & $\mathbf{E}$ \\
\hline 1 & 1 & -1 & -1 & -1 & -1 & 40 & 12 & 125 & 190 & 125 \\
\hline 2 & 1 & -1 & -1 & 1 & 1 & 40 & 12 & 125 & 210 & 135 \\
\hline 3 & -1 & 1 & 1 & 1 & -1 & 20 & 18 & 135 & 210 & 125 \\
\hline 4 & 1 & -1 & 1 & 1 & -1 & 40 & 12 & 135 & 210 & 125 \\
\hline 5 & -2 & 0 & 0 & 0 & 0 & 10 & 15 & 130 & 200 & 130 \\
\hline 6 & 0 & 0 & -2 & 0 & 0 & 30 & 15 & 120 & 200 & 130 \\
\hline 7 & -1 & 1 & -1 & 1 & 1 & 20 & 18 & 125 & 210 & 135 \\
\hline 8 & 2 & 0 & 0 & 0 & 0 & 50 & 15 & 130 & 200 & 130 \\
\hline 9 & 0 & 0 & 0 & 0 & 0 & 30 & 15 & 130 & 200 & 130 \\
\hline 10 & 0 & 0 & 2 & 0 & 0 & 30 & 15 & 140 & 200 & 130 \\
\hline 11 & -1 & -1 & 1 & 1 & 1 & 20 & 12 & 135 & 210 & 135 \\
\hline 12 & 1 & 1 & -1 & -1 & 1 & 40 & 18 & 125 & 190 & 135 \\
\hline 13 & 0 & 0 & 0 & 0 & 2 & 30 & 15 & 130 & 200 & 140 \\
\hline 14 & 0 & 0 & 0 & -2 & 0 & 30 & 15 & 130 & 180 & 130 \\
\hline 15 & 0 & 0 & 0 & 0 & 0 & 30 & 15 & 130 & 200 & 130 \\
\hline 16 & 0 & 0 & 0 & 0 & 0 & 30 & 15 & 130 & 200 & 130 \\
\hline 17 & 1 & 1 & 1 & -1 & -1 & 40 & 18 & 135 & 190 & 125 \\
\hline 18 & 0 & 0 & 0 & 0 & -2 & 30 & 15 & 130 & 200 & 120 \\
\hline 19 & 1 & -1 & 1 & -1 & 1 & 40 & 12 & 135 & 190 & 135 \\
\hline 20 & -1 & -1 & 1 & -1 & -1 & 20 & 12 & 135 & 190 & 125 \\
\hline 21 & 0 & 0 & 0 & 0 & 0 & 30 & 15 & 130 & 200 & 130 \\
\hline 22 & 0 & 0 & 0 & 0 & 0 & 30 & 15 & 130 & 200 & 130 \\
\hline 23 & -1 & -1 & -1 & 1 & -1 & 20 & 12 & 125 & 210 & 125 \\
\hline 24 & 0 & 0 & 0 & 0 & 0 & 30 & 15 & 130 & 200 & 130 \\
\hline 25 & 1 & 1 & 1 & 1 & 1 & 40 & 18 & 135 & 210 & 135 \\
\hline 26 & 0 & -2 & 0 & 0 & 0 & 30 & 9 & 130 & 200 & 130 \\
\hline 27 & -1 & -1 & -1 & -1 & 1 & 20 & 12 & 125 & 190 & 135 \\
\hline 28 & 0 & 2 & 0 & 0 & 0 & 30 & 21 & 130 & 200 & 130 \\
\hline 29 & -1 & 1 & 1 & -1 & 1 & 20 & 18 & 135 & 190 & 135 \\
\hline 30 & 1 & 1 & -1 & 1 & -1 & 40 & 18 & 125 & 210 & 125 \\
\hline 31 & 0 & 0 & 0 & 2 & 0 & 30 & 15 & 130 & 220 & 130 \\
\hline 32 & -1 & 1 & -1 & -1 & -1 & 20 & 18 & 125 & 190 & 125 \\
\hline
\end{tabular}

A:Blend Ratio (\%), B:Feed Moisture Content (\%), C:Barrel Temperature $\left({ }^{\circ} \mathrm{C}\right)$, D:Die Head Temperature $\left({ }^{\circ} \mathrm{C}\right)$, E:Screw Speed $(\mathrm{RPM})$ 
Int.J.Curr.Microbiol.App.Sci (2019) 8(10): $2164-2179$

Table.3 ANNOVA table for quadratic model of response surface for Crispiness

\begin{tabular}{|c|c|c|c|c|c|}
\hline Source & Sum of Squares & df & Mean Square & F-value & p-value \\
\hline Model & 142.20 & 20 & 7.11 & $3.330 \mathrm{E}+06$ & $<0.0001$ \\
\hline A-FMC & 3.76 & 1 & 3.76 & $1.761 \mathrm{E}+06$ & $<0.0001$ \\
\hline B-BR & 2.61 & 1 & 2.61 & $1.224 \mathrm{E}+06$ & $<0.0001$ \\
\hline C-DHT & 8.76 & 1 & 8.76 & $4.103 \mathrm{E}+06$ & $<0.0001$ \\
\hline D-BT & 23.05 & 1 & 23.05 & $1.080 \mathrm{E}+07$ & $<0.0001$ \\
\hline E-SS & 0.0337 & 1 & 0.0337 & 15808.06 & $<0.0001$ \\
\hline AB & 5.64 & 1 & 5.64 & $2.642 \mathrm{E}+06$ & $<0.0001$ \\
\hline $\mathbf{A C}$ & 0.6084 & 1 & 0.6084 & $2.850 \mathrm{E}+05$ & $<0.0001$ \\
\hline AD & 0.0006 & 1 & 0.0006 & 292.74 & $<0.0001$ \\
\hline $\mathbf{A E}$ & 9.46 & 1 & 9.46 & $4.429 \mathrm{E}+06$ & $<0.0001$ \\
\hline BC & 19.14 & 1 & 19.14 & $8.965 \mathrm{E}+06$ & $<0.0001$ \\
\hline BD & 2.02 & 1 & 2.02 & $9.445 \mathrm{E}+05$ & $<0.0001$ \\
\hline BE & 1.88 & 1 & 1.88 & $8.791 \mathrm{E}+05$ & $<0.0001$ \\
\hline CD & 5.88 & 1 & 5.88 & $2.754 \mathrm{E}+06$ & $<0.0001$ \\
\hline CE & 7.16 & 1 & 7.16 & $3.352 \mathrm{E}+06$ & $<0.0001$ \\
\hline DE & 0.0009 & 1 & 0.0009 & 421.55 & $<0.0001$ \\
\hline $\mathbf{A}^{2}$ & 8.72 & 1 & 8.72 & $4.084 \mathrm{E}+06$ & $<0.0001$ \\
\hline $\mathbf{B}^{2}$ & 39.12 & 1 & 39.12 & $1.832 \mathrm{E}+07$ & $<0.0001$ \\
\hline $\mathbf{C}^{2}$ & 0.0120 & 1 & 0.0120 & 5621.35 & $<0.0001$ \\
\hline$D^{2}$ & 0.7027 & 1 & 0.7027 & $3.291 \mathrm{E}+05$ & $<0.0001$ \\
\hline $\mathbf{E}^{2}$ & 0.8331 & 1 & 0.8331 & $3.902 \mathrm{E}+05$ & $<0.0001$ \\
\hline Residual & 0.0000 & 11 & $2.135 \mathrm{E}-06$ & & \\
\hline Lack of Fit & 0.0000 & 6 & $3.914 \mathrm{E}-06$ & & \\
\hline Pure Error & 0.0000 & 5 & 0.0000 & & \\
\hline Cor Total & 142.20 & 31 & & & \\
\hline
\end{tabular}

Table.4 ANNOVA table for quadratic model of response surface for Hardness

\begin{tabular}{|l|r|r|r|r|r|}
\hline Source & Sum of Squares & df & Mean Square & F-value & p-value \\
\hline Model & 412.96 & 20 & 20.65 & 1.47 & 0.2580 \\
\hline A-FMC & 0.2243 & 1 & 0.2243 & 0.0160 & 0.9017 \\
\hline B-BR & 3.60 & 1 & 3.60 & 0.2569 & 0.6223 \\
\hline C-DHT & 3.47 & 1 & 3.47 & 0.2471 & 0.6289 \\
\hline D-BT & 100.86 & 1 & 100.86 & 7.19 & 0.0214 \\
\hline E-SS & 48.56 & 1 & 48.56 & 3.46 & 0.0897 \\
\hline AB & 11.26 & 1 & 11.26 & 0.8024 & 0.3895 \\
\hline AC & 4.06 & 1 & 4.06 & 0.2895 & 0.6013 \\
\hline AD & 73.87 & 1 & 73.87 & 5.27 & 0.0424 \\
\hline AE & 7.45 & 1 & 7.45 & 0.5313 & 0.4813 \\
\hline BC & 105.58 & 1 & 105.58 & 7.53 & 0.0191 \\
\hline BD & 13.14 & 1 & 13.14 & 0.9368 & 0.3539 \\
\hline BE & 2.04 & 1 & 2.04 & 0.1458 & 0.7099 \\
\hline CD & 4.82 & 1 & 4.82 & 0.3435 & 0.5697 \\
\hline CE & 0.5625 & 1 & 0.5625 & 0.0401 & 0.8449 \\
\hline DE & 0.2304 & 1 & 0.2304 & 0.0164 & 0.9003 \\
\hline $\mathbf{A}^{\mathbf{2}}$ & 0.5985 & 1 & 0.5985 & 0.0427 & 0.8401 \\
\hline $\mathbf{B}^{2}$ & 5.45 & 1 & 5.45 & 0.3883 & 0.5459 \\
\hline $\mathbf{C}^{2}$ & 0.3856 & 1 & 0.3856 & 0.0275 & 0.8713 \\
\hline $\mathbf{D}^{\mathbf{2}}$ & 19.71 & 1 & 19.71 & 1.40 & 0.2609 \\
\hline $\mathbf{E}^{\mathbf{2}}$ & 10.37 & 1 & 10.37 & 0.7395 & 0.4082 \\
\hline Residual & 154.30 & 11 & 14.03 & & \\
\hline Lack of Fit & 33.94 & 6 & 5.66 & 0.2350 & 0.9467 \\
\hline Pure Error & 120.36 & 5 & 24.07 & & \\
\hline Cor Total & 567.26 & 31 & & & \\
\hline & & & & & \\
\hline
\end{tabular}


Table.5 ANNOVA table for quadratic model of response surface for Cutting Strength

\begin{tabular}{|c|c|c|c|c|c|}
\hline Source & $\begin{array}{c}\text { Sum of } \\
\text { Squares }\end{array}$ & df & $\begin{array}{c}\text { Mean } \\
\text { Square }\end{array}$ & F-value & p-value \\
\hline Model & 210.39 & 20 & 10.52 & 1.97 & 0.1248 \\
\hline A-FMC & 23.19 & 1 & 23.19 & 4.33 & 0.0615 \\
\hline B-BR & 71.52 & 1 & 71.52 & 13.36 & 0.0038 \\
\hline C-DHT & 0.4902 & 1 & 0.4902 & 0.0916 & 0.7678 \\
\hline D-BT & 5.99 & 1 & 5.99 & 1.12 & 0.3127 \\
\hline E-SS & 0.3567 & 1 & 0.3567 & 0.0667 & 0.8010 \\
\hline $\mathbf{A B}$ & 8.57 & 1 & 8.57 & 1.60 & 0.2318 \\
\hline $\mathbf{A C}$ & 2.65 & 1 & 2.65 & 0.4950 & 0.4963 \\
\hline AD & 15.43 & 1 & 15.43 & 2.88 & 0.1176 \\
\hline $\mathbf{A E}$ & 0.1914 & 1 & 0.1914 & 0.0358 & 0.8534 \\
\hline BC & 0.7439 & 1 & 0.7439 & 0.1390 & 0.7163 \\
\hline BD & 11.41 & 1 & 11.41 & 2.13 & 0.1722 \\
\hline BE & 6.46 & 1 & 6.46 & 1.21 & 0.2952 \\
\hline CD & 0.2233 & 1 & 0.2233 & 0.0417 & 0.8419 \\
\hline $\mathrm{CE}$ & 3.83 & 1 & 3.83 & 0.7160 & 0.4155 \\
\hline DE & 18.00 & 1 & 18.00 & 3.36 & 0.0938 \\
\hline $\mathbf{A}^{2}$ & 12.29 & 1 & 12.29 & 2.30 & 0.1578 \\
\hline $\mathbf{B}^{2}$ & 1.40 & 1 & 1.40 & 0.2619 & 0.6189 \\
\hline $\mathrm{C}^{2}$ & 0.8618 & 1 & 0.8618 & 0.1611 & 0.6959 \\
\hline $\mathbf{D}^{2}$ & 15.27 & 1 & 15.27 & 2.85 & 0.1193 \\
\hline $\mathbf{E}^{2}$ & 8.67 & 1 & 8.67 & 1.62 & 0.2293 \\
\hline Residual & 58.87 & 11 & 5.35 & & \\
\hline Lack of Fit & 32.18 & 6 & 5.36 & 1.00 & 0.5085 \\
\hline Pure Error & 26.69 & 5 & 5.34 & & \\
\hline Cor Total & 269.25 & 31 & & & \\
\hline
\end{tabular}

Table.6 Regression coefficient of second order mathematical model for Crispness of extrudates

\begin{tabular}{|l|r|r|r|r|r|r|}
\hline \multicolumn{1}{|c|}{ Factor } & $\begin{array}{c}\text { Coefficient } \\
\text { Estimate }\end{array}$ & df & $\begin{array}{c}\text { Standard } \\
\text { Error }\end{array}$ & 95\% CI Low & \multicolumn{1}{c|}{$\begin{array}{c}\text { 95\% CI } \\
\text { High }\end{array}$} & VIF \\
\hline Intercept & 15.49 & 1 & 0.4697 & 14.46 & 16.52 & \\
\hline A-FMC & -0.1767 & 1 & 0.2404 & -0.7057 & 0.3523 & 1.0000 \\
\hline B-BR & -0.8675 & 1 & 0.2404 & -1.40 & -0.3385 & 1.0000 \\
\hline C-DHT & 0.1233 & 1 & 0.2404 & -0.4057 & 0.6523 & 1.0000 \\
\hline D-BT & 0.3775 & 1 & 0.2404 & -0.1515 & 0.9065 & 1.0000 \\
\hline E-SS & 0.1067 & 1 & 0.2404 & -0.4223 & 0.6357 & 1.0000 \\
\hline AB & 0.2525 & 1 & 0.2944 & -0.3954 & 0.9004 & 1.0000 \\
\hline AC & 0.1388 & 1 & 0.2944 & -0.5092 & 0.7867 & 1.0000 \\
\hline AD & -0.2150 & 1 & 0.2944 & -0.8629 & 0.4329 & 1.0000 \\
\hline AE & 0.1525 & 1 & 0.2944 & -0.4954 & 0.8004 & 1.0000 \\
\hline BC & 0.3025 & 1 & 0.2944 & -0.3454 & 0.9504 & 1.0000 \\
\hline BD & 0.2713 & 1 & 0.2944 & -0.3767 & 0.9192 & 1.0000 \\
\hline BE & -0.1113 & 1 & 0.2944 & -0.7592 & 0.5367 & 1.0000 \\
\hline CD & -0.2650 & 1 & 0.2944 & -0.9129 & 0.3829 & 1.0000 \\
\hline CE & 0.0525 & 1 & 0.2944 & -0.5954 & 0.7004 & 1.0000 \\
\hline DE & 0.1462 & 1 & 0.2944 & -0.5017 & 0.7942 & 1.0000 \\
\hline $\mathbf{A}^{2}$ & 0.0741 & 1 & 0.2174 & -0.4044 & 0.5526 & 1.02 \\
\hline $\mathbf{B}^{2}$ & -0.8759 & 1 & 0.2174 & -1.35 & -0.3974 & 1.02 \\
\hline $\mathbf{C}^{2}$ & -0.2009 & 1 & 0.2174 & -0.6794 & 0.2776 & 1.02 \\
\hline $\mathbf{D}^{2}$ & -0.3759 & 1 & 0.2174 & -0.8544 & 0.1026 & 1.02 \\
\hline $\mathbf{E}^{2}$ & -0.3897 & 1 & 0.2174 & -0.8682 & 0.0889 & 1.02 \\
\hline
\end{tabular}


Table.7Regression coefficient of second order mathematical model for Hardness of extrudates

\begin{tabular}{|l|l|l|l|l|l|l|}
\hline Factor & Coefficient Estimate & df & Standard Error & $\mathbf{9 5 \%}$ CI Low & $\mathbf{9 5 \%}$ CI High & VIF \\
\hline Intercept & 10.12 & 1 & 1.49 & 6.83 & 13.40 & \\
\hline A-FMC & 0.0967 & 1 & 0.7645 & -1.59 & 1.78 & 1.0000 \\
\hline B-BR & 0.3875 & 1 & 0.7645 & -1.30 & 2.07 & 1.0000 \\
\hline C-DHT & -0.3800 & 1 & 0.7645 & -2.06 & 1.30 & 1.0000 \\
\hline D-BT & -2.05 & 1 & 0.7645 & -3.73 & -0.3673 & 1.0000 \\
\hline E-SS & 1.42 & 1 & 0.7645 & -0.2602 & 3.11 & 1.0000 \\
\hline AB & -0.8388 & 1 & 0.9363 & -2.90 & 1.22 & 1.0000 \\
\hline AC & 0.5037 & 1 & 0.9363 & -1.56 & 2.56 & 1.0000 \\
\hline AD & -2.15 & 1 & 0.9363 & -4.21 & -0.0879 & 1.0000 \\
\hline AE & 0.6825 & 1 & 0.9363 & -1.38 & 2.74 & 1.0000 \\
\hline BC & -2.57 & 1 & 0.9363 & -4.63 & -0.5079 & 1.0000 \\
\hline BD & -0.9063 & 1 & 0.9363 & -2.97 & 1.15 & 1.0000 \\
\hline BE & -0.3575 & 1 & 0.9363 & -2.42 & 1.70 & 1.0000 \\
\hline CD & -0.5488 & 1 & 0.9363 & -2.61 & 1.51 & 1.0000 \\
\hline CE & -0.1875 & 1 & 0.9363 & -2.25 & 1.87 & 1.0000 \\
\hline DE & -0.1200 & 1 & 0.9363 & -2.18 & 1.94 & 1.0000 \\
\hline $\mathbf{A}^{\mathbf{2}}$ & -0.1428 & 1 & 0.6915 & -1.66 & 1.38 & 1.02 \\
\hline $\mathbf{B}^{\mathbf{2}}$ & 0.4309 & 1 & 0.6915 & -1.09 & 1.95 & 1.02 \\
\hline $\mathbf{C}^{\mathbf{2}}$ & 0.1147 & 1 & 0.6915 & -1.41 & 1.64 & 1.02 \\
\hline $\mathbf{D}^{\mathbf{2}}$ & 0.8197 & 1 & 0.6915 & -0.7024 & 2.34 & 1.02 \\
\hline $\mathbf{E}^{\mathbf{2}}$ & 0.5947 & 1 & 0.6915 & -0.9274 & 2.12 & 1.02 \\
\hline
\end{tabular}

Table.8 Regression coefficient of second order mathematical model for Cutting Strength of extrudates

\begin{tabular}{|l|r|r|r|r|r|r|}
\hline Factor & $\begin{array}{c}\text { Coefficient } \\
\text { Estimate }\end{array}$ & df & $\begin{array}{c}\text { Standard } \\
\text { Error }\end{array}$ & $\begin{array}{c}\text { 95\% CI } \\
\text { Low }\end{array}$ & $\begin{array}{c}\text { 95\% CI } \\
\text { High }\end{array}$ & \multicolumn{1}{c|}{ VIF } \\
\hline Intercept & 9.35 & 1 & 0.9227 & 7.32 & 11.38 & \\
\hline A-FMC & 0.9829 & 1 & 0.4722 & -0.0564 & 2.02 & 1.0000 \\
\hline B-BR & 1.73 & 1 & 0.4722 & 0.6869 & 2.77 & 1.0000 \\
\hline C-DHT & 0.1429 & 1 & 0.4722 & -0.8964 & 1.18 & 1.0000 \\
\hline D-BT & 0.4996 & 1 & 0.4722 & -0.5397 & 1.54 & 1.0000 \\
\hline E-SS & -0.1219 & 1 & 0.4722 & -1.16 & 0.9174 & 1.0000 \\
\hline AB & 0.7319 & 1 & 0.5783 & -0.5410 & 2.00 & 1.0000 \\
\hline AC & 0.4069 & 1 & 0.5783 & -0.8660 & 1.68 & 1.0000 \\
\hline AD & 0.9819 & 1 & 0.5783 & -0.2910 & 2.25 & 1.0000 \\
\hline AE & 0.1094 & 1 & 0.5783 & -1.16 & 1.38 & 1.0000 \\
\hline BC & 0.2156 & 1 & 0.5783 & -1.06 & 1.49 & 1.0000 \\
\hline BD & -0.8444 & 1 & 0.5783 & -2.12 & 0.4285 & 1.0000 \\
\hline BE & 0.6356 & 1 & 0.5783 & -0.6373 & 1.91 & 1.0000 \\
\hline CD & 0.1181 & 1 & 0.5783 & -1.15 & 1.39 & 1.0000 \\
\hline CE & -0.4894 & 1 & 0.5783 & -1.76 & 0.7835 & 1.0000 \\
\hline DE & 1.06 & 1 & 0.5783 & -0.2123 & 2.33 & 1.0000 \\
\hline $\mathbf{A}^{\mathbf{2}}$ & 0.6473 & 1 & 0.4271 & -0.2927 & 1.59 & 1.02 \\
\hline $\mathbf{B}^{2}$ & 0.2186 & 1 & 0.4271 & -0.7215 & 1.16 & 1.02 \\
\hline $\mathbf{C}^{2}$ & -0.1714 & 1 & 0.4271 & -1.11 & 0.7687 & 1.02 \\
\hline $\mathbf{D}^{\mathbf{2}}$ & -0.7214 & 1 & 0.4271 & -1.66 & 0.2187 & 1.02 \\
\hline $\mathbf{E}^{\mathbf{2}}$ & -0.5437 & 1 & 0.4271 & -1.48 & 0.3964 & 1.02 \\
\hline
\end{tabular}




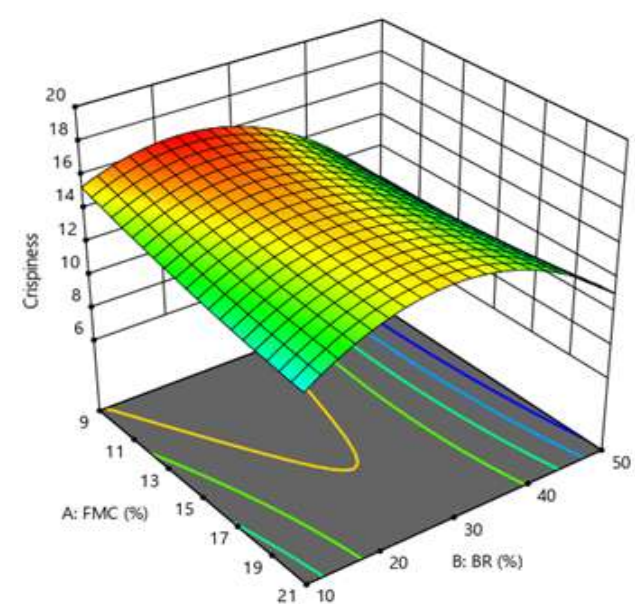

Fig.1 Effect of feed moisture content and blend ratio on crispness of extrudates

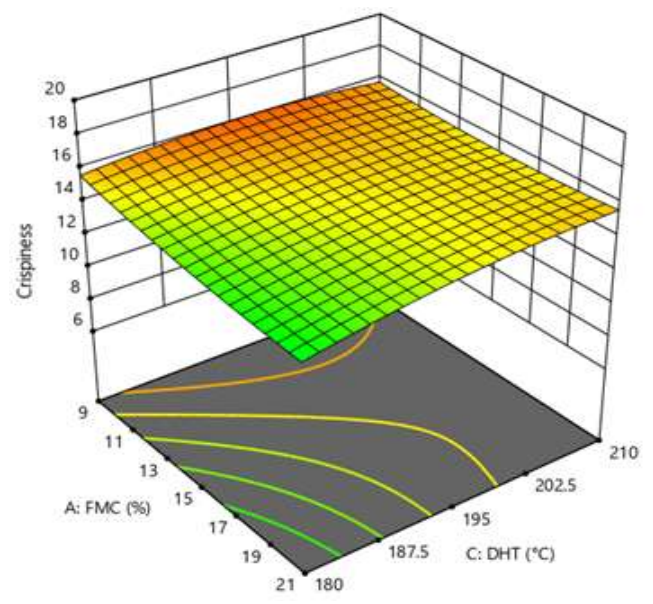

Fig.3 Effect of feed moisture content and die head temperature on crispness of extrudates

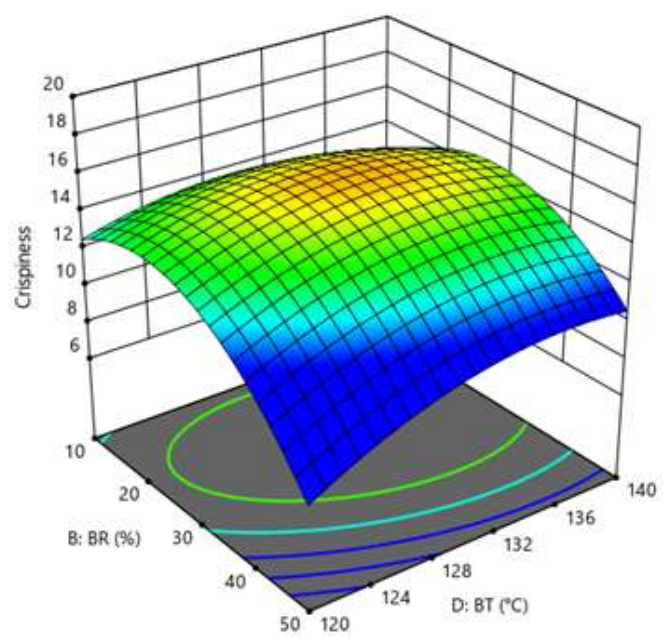

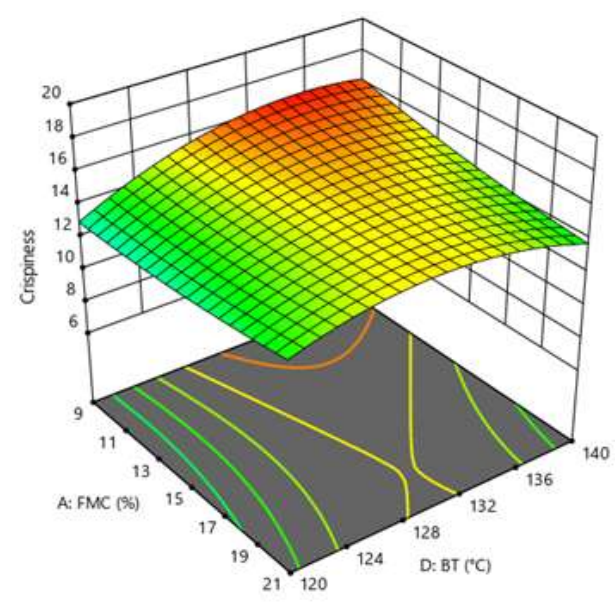

Fig.2 Effect of feed moisture content and barrel temperature on crispness of extrudates

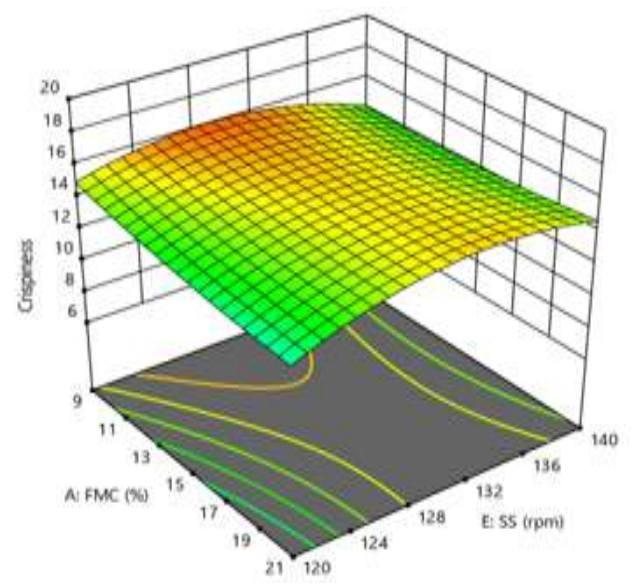

Fig.4 Effect of feed moisture content and screw speed on crispness of extrudates

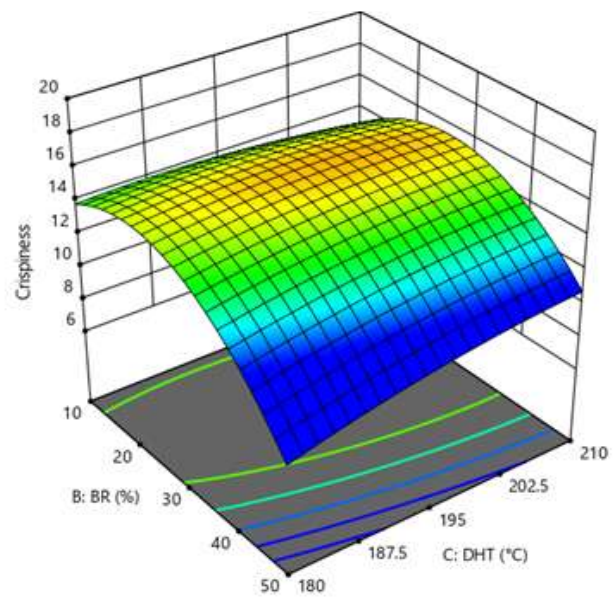


Fig.5 Effect of blend ratio and barrel temperature on crispness of extrudates

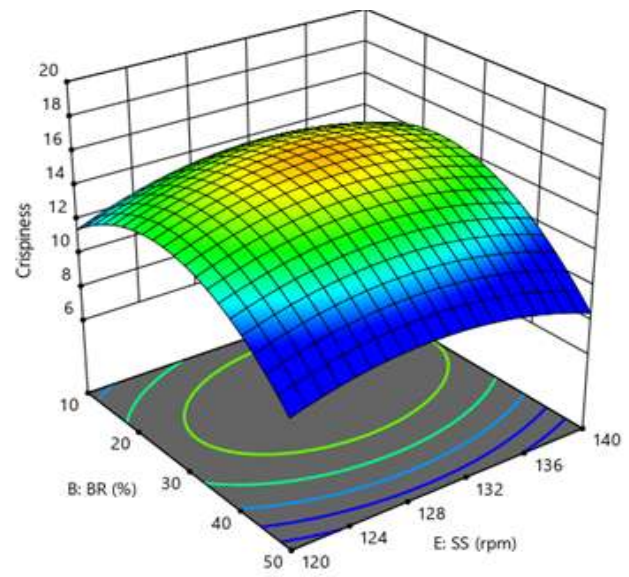

Fig.7 Effect of blend ratio and screw speed crispness of extrudates

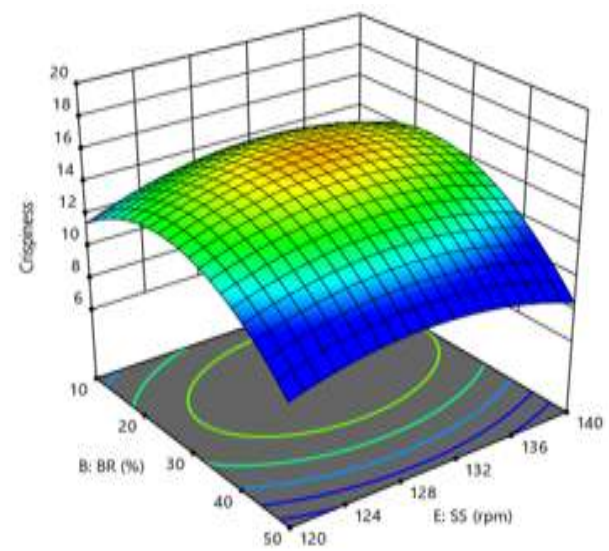

Fig.9 Effect of barrel temperature and screw speed on crispness of extrudates

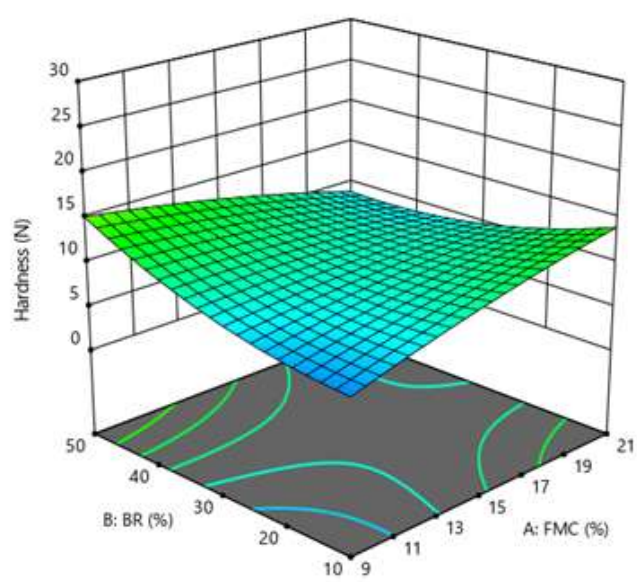

Fig.6 Effect of blend ratio and die head temperature on crispness of extrudates

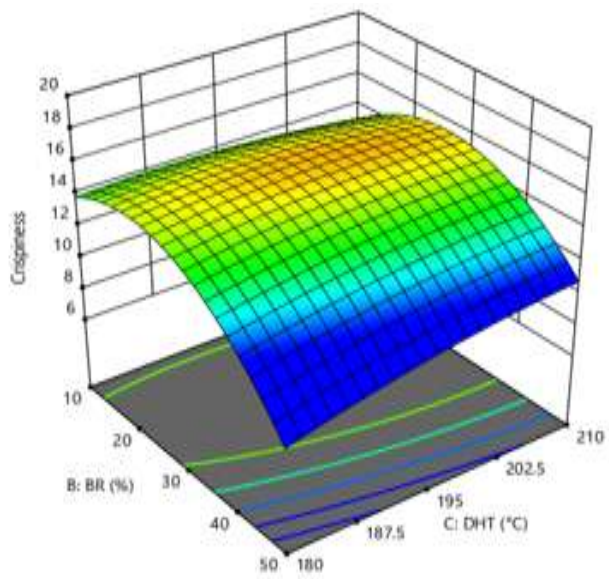

Fig.8 Effect of barrel temperature and die head temperature on crispness of extrudates

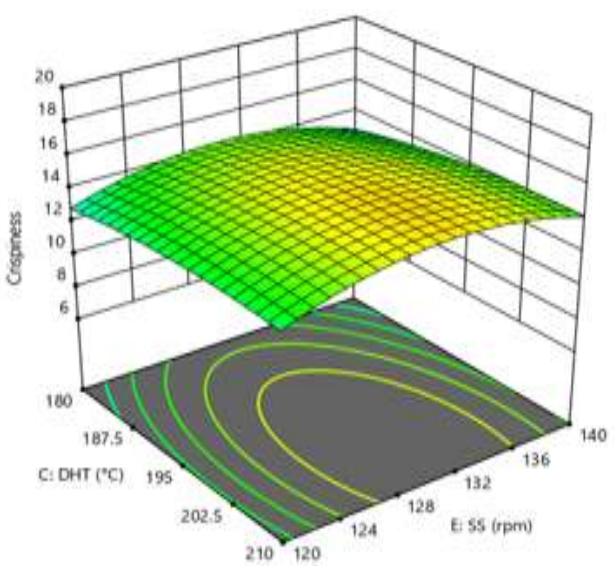

Fig.10 Effect of die head temperature and screw speed on crispness of extrudates

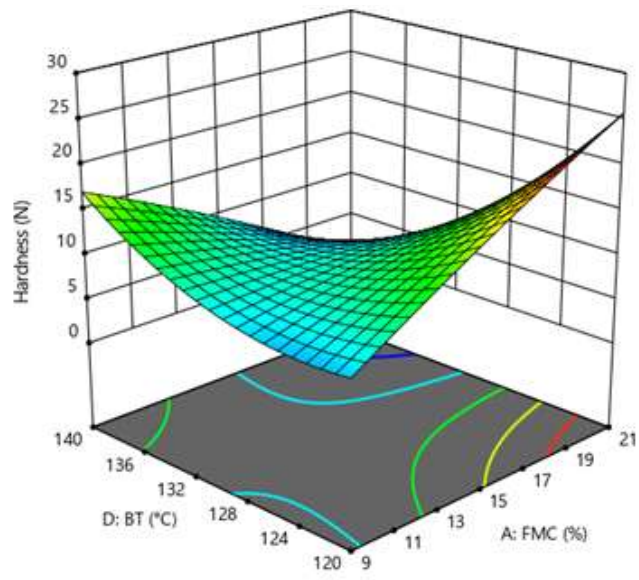


Fig.11 Effect of feed moisture content and blend ratio on hardness of extrudates

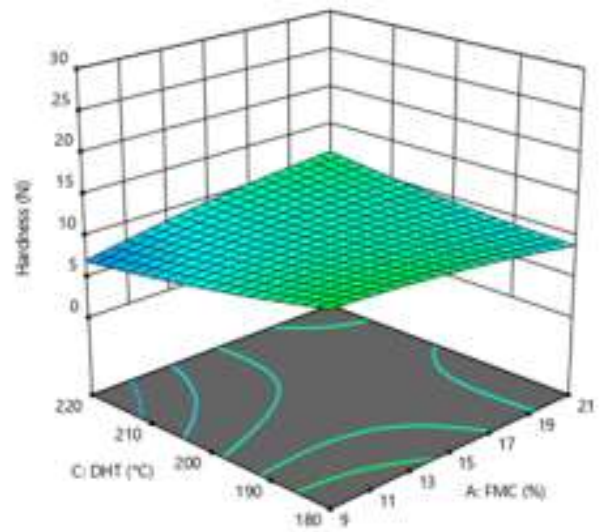

Fig.13 Effect of feed moisture content and die head temperature on hardness of extrudates

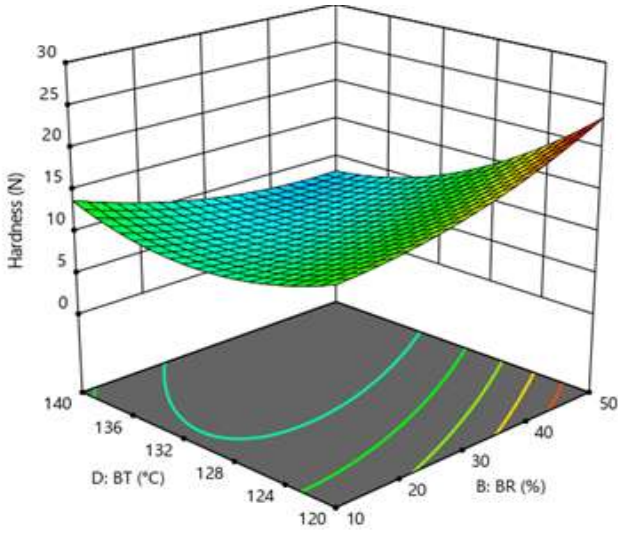

Fig.15 Effect of blend ratio and barrel temperature on hardness of extrudates

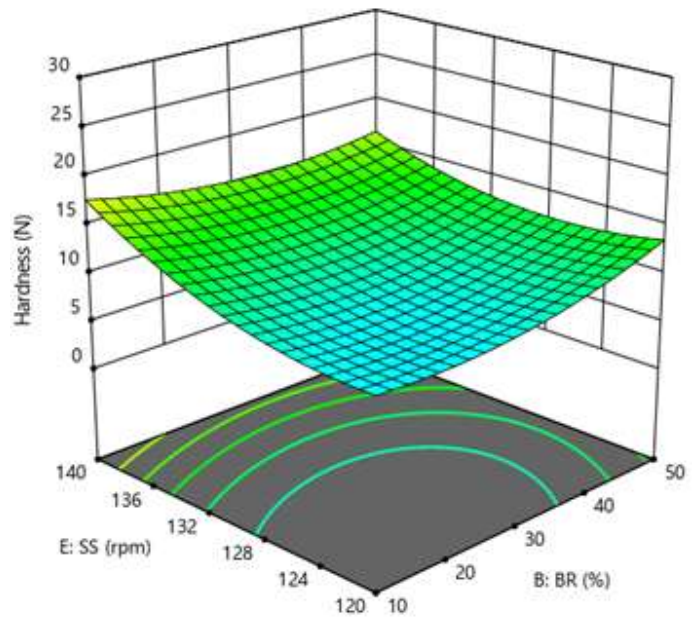

Fig.12 Effect of feed moisture content and barrel temperature on hardness of extrudates

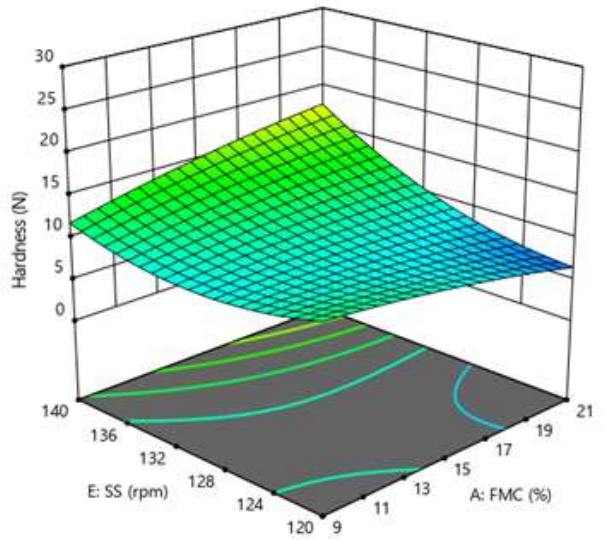

Fig.14 Effect of feed moisture content and screw speed on hardness of extrudates

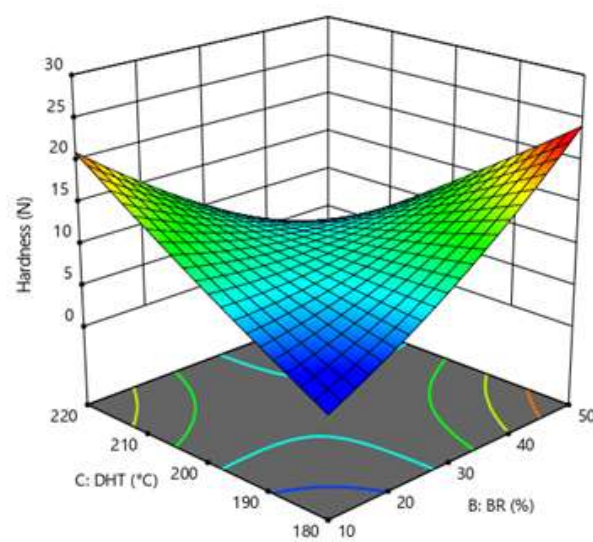

Fig.16 Effect of blend ratio and die head temperature on hardness of extrudates

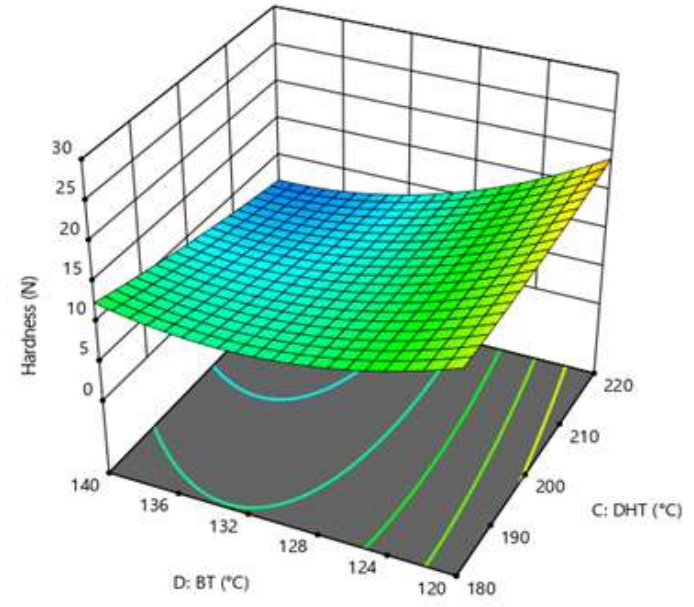


Fig.17 Effect of blend ratio and screw speed on hardness of extrudates

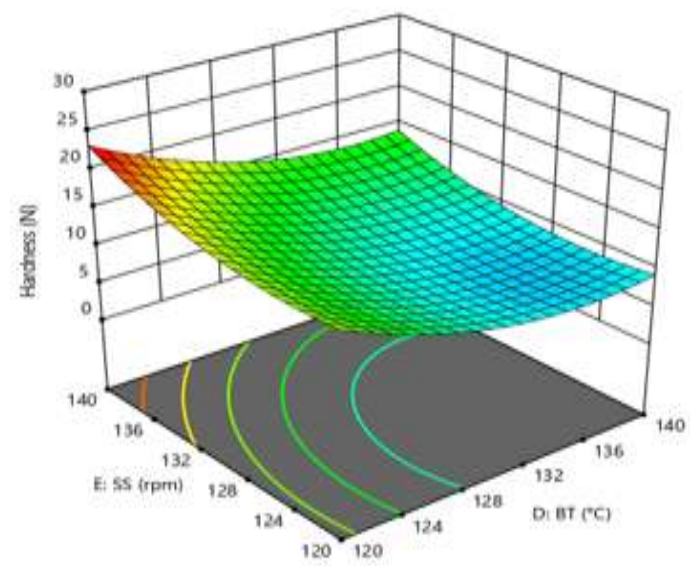

Fig.19 Effect of barrel temperature and screw speed on hardness of extrudates

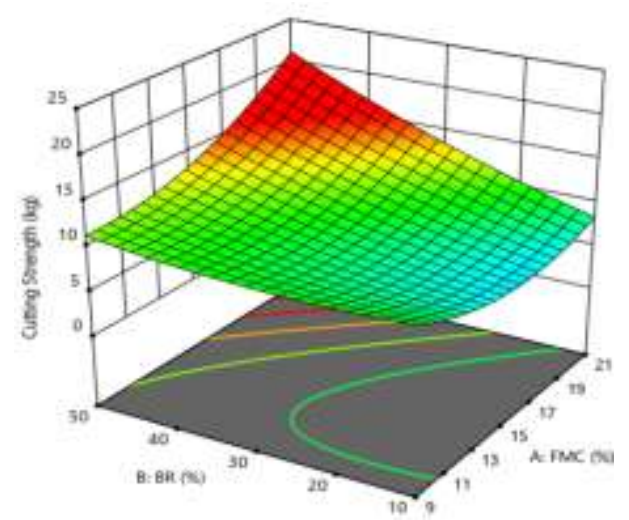

Fig.21 Effect of feed moisture content and blend ratio cutting strength of extrudates

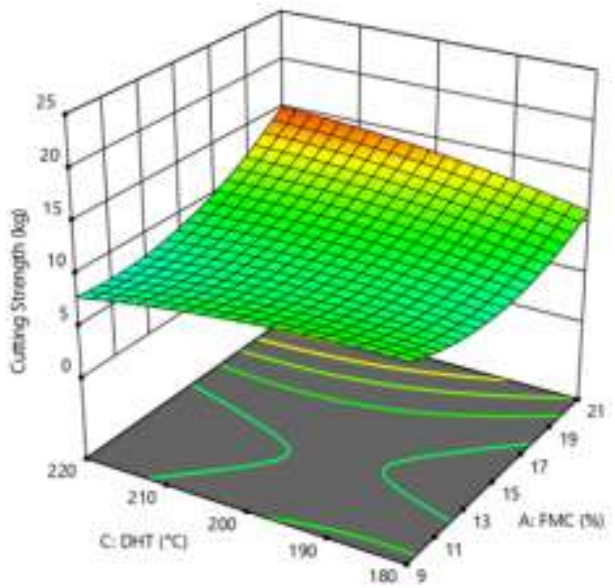

Fig.18 Effect of barrel temperature and die head temperature on hardness of extrudates

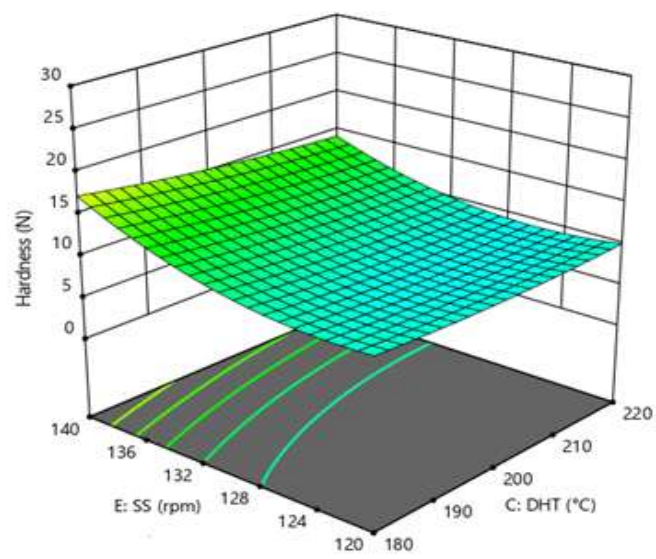

Fig.20 Effect of die head temperature and screw speed on hardness of extrudates

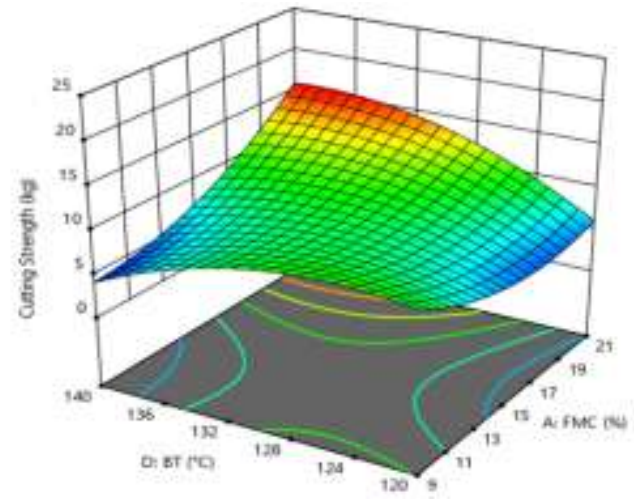

Fig.22 Effect of feed moisture content and barrel temperature on cutting strength of extrudates

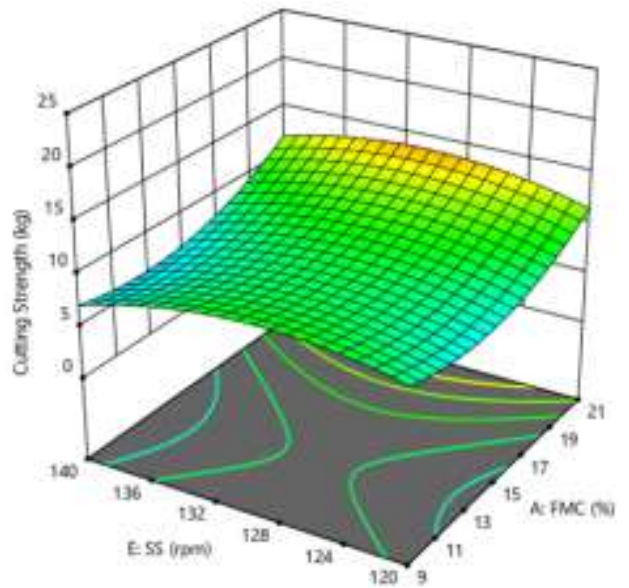


Fig.23 Effect of feed moisture content and die head temperature on cutting strength of extrudates

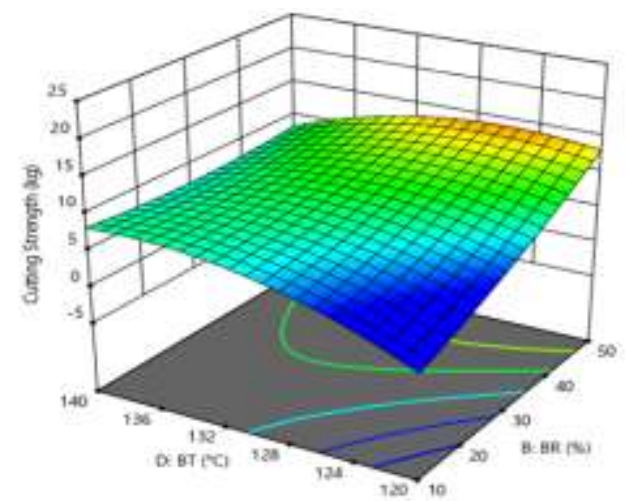

Fig.25 Effect of blend ratio and barrel temperature on cutting strength of extrudates

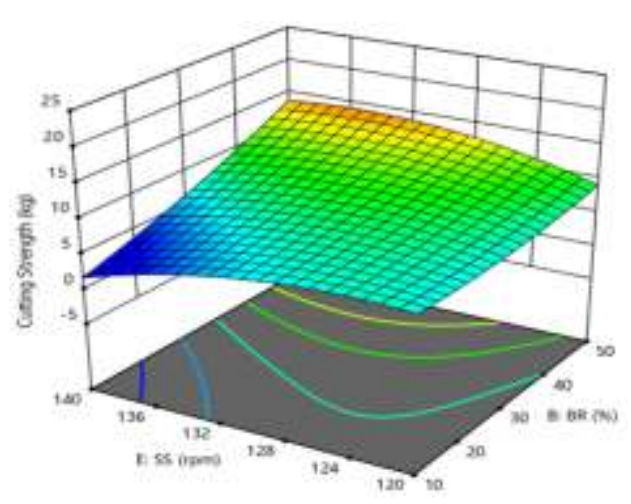

Fig.27 Effect of blend ratio and screw speed on cutting strength of extrudates

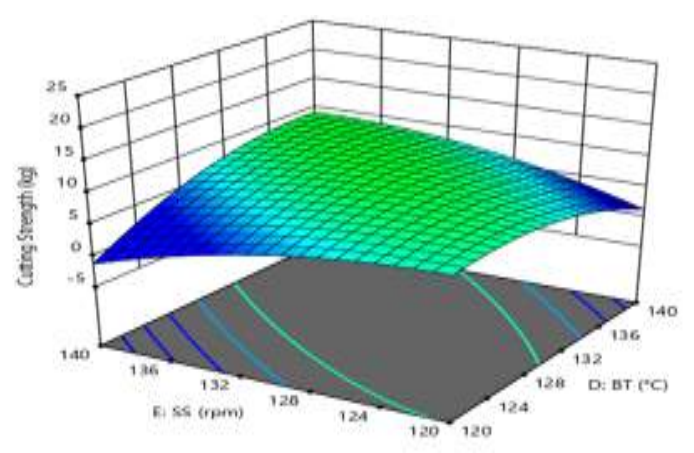

Fig.29 Effect of barrel temperature and screw speed on cutting strength of extrudates
Fig.24 Effect of feed moisture content and screw speed on cutting strength of extrudates

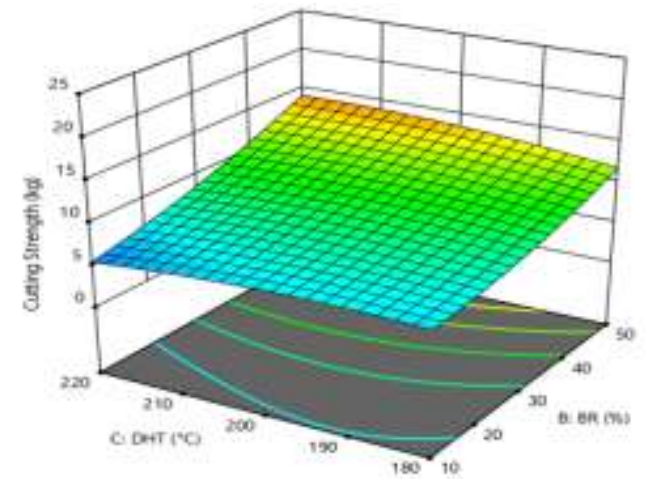

Fig.26 Effect of blend ratio and die head temperature on cutting strength of extrudates

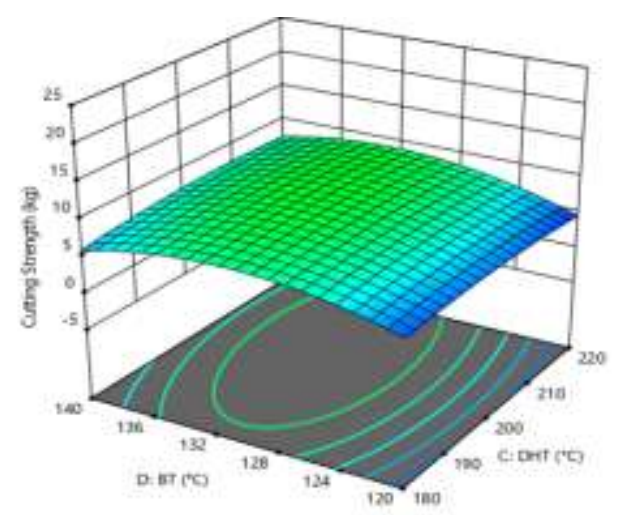

Fig.28 Effect of barrel temperature and die head temperature on cutting strength of extrudates

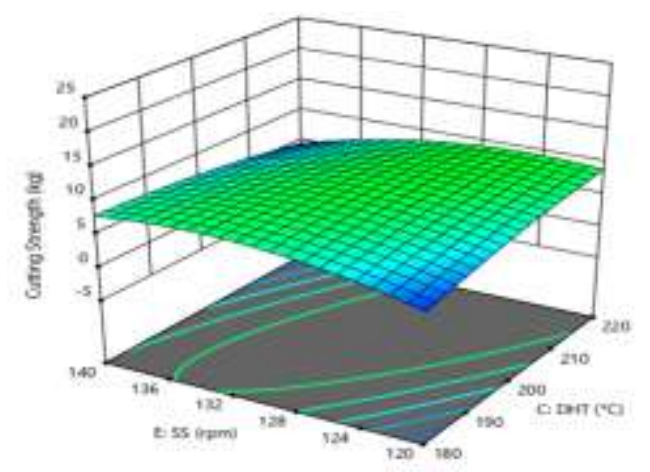

Fig.30 Effect of die head temperature and screw speed on cutting strength of extrudates 
It is observed that when moisture content of feed and blend ratio increased (increasing protein content and decreasing starch), cutting strength of extrudate also increased. It may be because the feed materials became hard due to increasing moisture content of feeding. Further it was observed that barrel temperature and screw speed increased gradually, the cutting strength of extrudate decreased. This may be because more moisture was evaporated at high temperature causing formation of bubbles and consequently puffing. Therefore cutting strength reduced.

Effects of process and operational parameters on extrudate were shown in Figures 21-30. Minimum value of cutting strength of extrudate observed at $18 \%$ feed moisture content, 80:20 of blend ratio, $135 \mathrm{rpm}$ screw speed, $130{ }^{0} \mathrm{C}$ barrel temperature and $210{ }^{\circ} \mathrm{C}$ die head temperature

In conclusion, rice and pigeon pea are grown in appreciable amount in most of the part of India; the traditional milling process yields significant amounts in the form of brokens from rice and dal mill industries. That brokens do not find appropriate market and are listed as losses of the milling industries, generally disposed off cheaply, only to be used as animal feed or to acts as raw material for manufacturing other complementary products. The broken part of rice and pulses, are equally rich in the nutrition as comparable to whole grain. Moreover, high protein byproduct of industry offer scope for their use as supplement but all these require heat processing to make them suitable for human consumption. Extrusion cooking is one of the most important food processing technologies which have been used for the production of breakfast cereals, ready to eat snack foods and other textured foods. Effects of extrusion cooking on texture are ambiguous. As a complex multivariate process, extrusion cooking requires careful control if product quality is to be maintained. In this study it has to be mainly focused on the relationships between process and operation parameters on textural quality of extrudates which were prepared by flour of rice and dal milling industry's by-products i.e. brokens. The crispness of the extrudates varied between 11.31 and 16.66. The hardness of the extrudates varied between 4.63 and $22.30 \mathrm{~N}$ whereas the cutting strength of extrudate varied from 4.07 to $15.86 \mathrm{~kg}$. Minimum value of hardness and cutting strength of extrudate observed at $15 \%$ and $18 \%$ moisture content of feed respectively, 80:20 of feed blend ratio, $135{ }^{0} \mathrm{C}$ barrel temperature $190{ }^{0} \mathrm{C}$ die head temperature and $135 \mathrm{rpm}$ screw speed.

\section{References}

Adekola K A. (2014). Analytical engineering designs for twin screw food extruder dies. International Journal of Engineering Innovation and Research 3(5): 713-717.

Ahmed S M S, Zhang Q, Chen J and Shen Q. (2013). Millets Grains: Nutritional Quality, Processing and Potential Health Benefits. Comprehensive Reviews in Food Science and Food Safety 12: 281-295.

Anderson, R.A., Conway, H.F., Pfeifer, V.F., Griffin, Jr. E.1. (1969). Gelatinization of corn grits by roll and extrusion cooking. Cereal Science Today, 14(4-7),11-12.

Badrie, N., Mellowes, W. A. (1991). Texture and microstructure of cassava flour extrudate. Journal of Food Science, 56, 1319-1322, 1364.

Balandran- quantana, R.R., Barbosa, J.J., Zazueta, J.J., Anzaldua, A., Quinterro, A. (1998). Fuctional and nutritional properties of extruded of whole pinto bean meal (Phaseolus vulgaris 1.). Journal of Food Science, 63(1), 113116. 
Balasubramanian S, Singh K K, Patil RT and Onkar K K. (2012). Quality evaluation of millet-soy blended extrudates formulated through linear programming. J Food Sci Technol., 49(4): 450-458

Chen L and Opara U L. (2013). Texture measurement approach in fresh and processed foods - A review. Food Research International 51(2): 823-835.

Cochran, W.G., Cox, G.M. (1957). A text book of experimental designs. Publs. John Wiley \& Sons. Inc.:335-375

De Cindio B, Gabriele D, Pollini CM, Peressini D, Sensidoni A (2002). Filled snack production by extrusion-cooking: Rheological modelling of the process. J Food Eng 52: 67-74

Ding Q-B, Paul Ainsworth, Andrew Plunkett, Gregory Tucker and Hayley Marson (2006). The effect of extrusion conditions on the functional and physical properties of wheat-based expanded snacks. J Food Eng 73(2): 142-148

Deshpande H W and Poshadri A. (2011). Physical and sensory characteristics of extruded snacks prepared from Foxtail millet based composite flours. International Food Research Journal 18: 751-756.

Harper J M 1981. Extrusion of Foods, Baca Raton, CRC: 212
IASRI (2016) Production and productivity in agriculture during past 50 years. In: Agriculture research Data Book 2016. Indian Agriculture Statistical Research Institute. URL: http://www.iasri.res.in.

Liu Y, Hsieh F, Heymann H, Huff HE 2000. Effect of process conditions on the physical and sensory properties of extruded oat-corn puff. Journal of Food Science 65:1253-1259.

Myers R H, Montgomery D C and AndersonCook CM. (2008). Response surface methodology Process and Product Optimization Using Designed Experiments 3rd Edition. Publ. John Willey and Sons Inc. New Jersey. $\mathrm{p} 1$.

Shah Abhay Arvind (2003). The effect of extrusion conditions on aggregation of peanut proteins, Published M.S. Thesis, Submitted to the Graduate Faculty of The University of Georgia, Athens, Georgia.

Stanley D W. (1986). Chemical and structural determinants of texture of fabricated foods. Food Technology 40(3): 65-68, 76

Tosh S M and Yada S. (2010). Dietary fibres in pulse seeds and fractions: Characterization, functional attributes, and applications. Food Research International 43(2): 450-460.

\section{How to cite this article:}

Ajeet Sarathe, Ashwani Duggal and Mohan Singh. 2019. Development of Model for Extrusion Cooking Based on Textural Properties of Extrudate Prepared from Rice Broken and Pigeon Pea Dal Broken Flour Blends. Int.J.Curr.Microbiol.App.Sci. 8(10): 2164-2179. doi: https://doi.org/10.20546/ijcmas.2019.810.252 